\section{Third Virial Coefficients for Helium}

\section{R. E. Caligaris *}

Facultad Regional San Nicolás, Universidad Tecnológica Nacional, San Nicolás, Argentina

and

\section{J. C. Grangel}

Departamento de Astronomía y Física, Facultad de Humanidades y Ciencias, Universidad de la República, Cerrito 73, Montevideo, Uruguay

Z. Naturforsch. 33a, 1095-1096 (1978); received May 30,1978

Third virial coefficient for helium is calculated using the realistic Beck pair potential. The first quantum correction and the triple-dipole dispersion interaction are included. The results are compared with experimental data and with theoretical values obtained using the MDD-2 pair potential.

Some years ago we published [1] a computation of the third virial coefficient for helium using the MDD-2 pair potential proposed by Bruch and McGee [2]. We included the first quantum correction and the contribution due to the triple-dipole dispersion interaction. We planned to extend our calculations using the pair potential proposed by Beck [3]. But simultaneously Ram and Singh [4]

* Member of the Carrera del Investigador Científico of the Consejo Nacional de Investigaciones Científicas y Técnicas. Argentina.

Reprint requests to R. E. Caligaris, Facultad Regional San Nicolás, Universidad Tecnológica Nacional C. C. 118 2900 San Nicolás, Argentinien. published similar computations using the LennardJones pair potential for helium and suggested that further improvement in the calculated values of the third virial coefficient could be achieved by using a more realistic pair potential such as that of Beck. So we stopped our calculations.

As we are not aware of calculations of the third virial coefficient using the Beck potential since, we now give our results for that potential.

Since we consider this work as a complement of the previous one [1] we are giving directly the equations used and the results obtained. The third virial coefficient $B_{3}(T)$ is given by

$$
\begin{aligned}
B_{3}(T)=B_{3}(\mathrm{ad}) & +\Delta B_{3}(\mathrm{ddd}) \\
& +\Lambda^{* 2} B_{3}(\mathrm{I}),
\end{aligned}
$$

where $B_{3}(\mathrm{ad})$ is the classical additive third virial coefficient, $\Delta B_{3}$ (ddd) is the classical non-additive third virial coefficient where the triple-dipole dispersion interaction [5] is used, and $B_{3}(\mathrm{I})$ is the first quantum correction to the third virial coefficient. The quantum correction to the non-additive third virial coefficient is negligible. The little contribution due to the triple-dipole dispersion interaction [1] makes the contribution due to other triple interactions negligible.

The Beck potential is given by:

$$
\begin{aligned}
v(r)= & A \exp \left\{-\alpha r-\beta r^{6}\right\} \\
& -\frac{C_{6}}{\left(r^{2}+a^{2}\right)^{3}}\left\{1+\frac{2.709+3 a^{2}}{r^{2}+a^{2}}\right\} .
\end{aligned}
$$

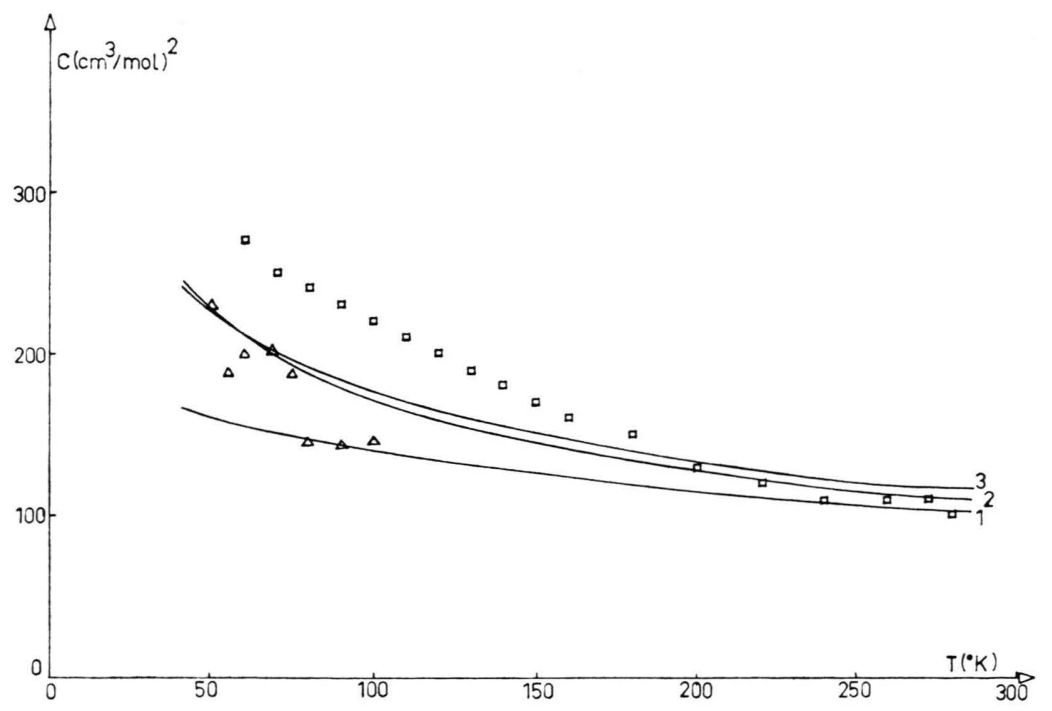

Fig. 1. The third virial coefficient $B_{3}$ of helium plotted against the absolute temperature $T$. Curve 1: $B_{3}(\mathrm{ad})$ obtained using Beck's potential; curve 2 : $B_{3}$ (classical additive + classical ddd term + first quantum correction) obtained using Beck's potential; curve $3: B_{3}$ (classical additive + classical ddd term + first quantum correction) obtained using MDD-2 potential. Experiment data: $\square$ ref.(6); $\triangle$ ref. (7). 
The well depth $\varepsilon$ of the potential is $1.43 \times 10^{-22} \mathrm{~J}$ at a distance $r_{\mathrm{m}}=2.969 \times 10^{-10} \mathrm{~m}$.

In Fig. 1 the theoretical values for the third virial coefficients are compared with the experimental ones. The values for the third virial coefficients computed with the MDD-2 pair potential are also shown. It is seen that there is not much differences between the results obtained using realistic pair potentials such as the MDD-2 or Beck's potential.

[1] R. E. Caligaris and J. C. Grangel, Chem. Phys. 2, 249 (1973).

[2] L. W. Bruch and I. J. McGee, J. Chem. Phys. 52, 5884 (1970).

[3] D. E. Beck, Molec. Phys. 14, 311 (1968).

[4] J. Ram and Y. Singh, Molec. Phys. 26, 539 (1973).
Our final comment in the discussion of the previous work [1] remains still valid.

\section{Acknowledgement}

The support provided by the Organization of American States through the "Programa Regional de Desarrollo Científico y Tecnológico" is gratefully acknowledged.

[5] R. J. Bell, J. Phys. B3, 751 (1970).

[6] J. M. H. Levelt Sengers, M. Klein, and J. S. Gallagher, AIP Handbook (1972).

[7] D. White, T. Rubin, P. Camky, and H. L. Johnston, J. Phys. Chem. 64, 1607 (1960). 